\title{
Estado nutricional de adultos atendidos em Estratégias de Saúde da Família de um município do Vale do Paranhana - RS
}

\author{
Nutritional Status of Adults Assisted in Family Health \\ Strategies of a Municipality of Vale do Paranhana - RS
}

Tamiris Gottlieb', Claudia Winter

'Universidade Feevale

E-mail para contato: Tamiris Gottlieb - tamirisgottlieb@hotmail.com

\begin{abstract}
Resumo
Os objetivos deste estudo foram avaliar o estado nutricional, consumo alimentar e identificar as patologias mais prevalentes de adultos atendidos em Estratégias de Saúde da Família (ESF). Foi realizado um estudo com delineamento transversal, observacional e de abordagem quantitativa. Os parâmetros antropométricos avaliados foram peso, altura e circunferência da cintura, e aplicação de dois questionários, um sobre dados socioeconômicos e clínicos e outro sobre consumo alimentar. Os dados foram analisados por meio do software SPSS versão 25.0 e as variáveis foram relacionadas através do teste Qui-quadrado de Pearson. Foram avaliados 180 adultos de ambos os sexos, destes $29,4 \%$ estavam com sobrepeso e $42,8 \%$ com obesidade, houve prevalência de doenças crônicas, principalmente hipertensão arterial sistêmica (HAS) e elevada tendência para o consumo de alimentos doces. Houve associação significativa entre o diagnóstico nutricional de obesidade e hipertensão arterial sistêmica, diabetes mellitus, dislipidemias, depressão, adiposidade abdominal e uso de medicações. Não houve associação significativa entre o consumo alimentar e obesidade. O estudo demonstrou elevada prevalência de excesso de peso (sobrepeso e de obesidade), de doenças crônicas não transmissíveis e consumo de alimentos ultraprocessados doces, sendo necessárias estratégias de enfrentamento a essas comorbidades.
\end{abstract}

Palavras-chave: Estado Nutricional. Estratégia Saúde da Família. Excesso de Peso. Obesidade. Doenças Crônicas Não Transmissíveis.

\begin{abstract}
The objectives of this study were to evaluate nutritional status, food consumption and to identify the most prevalent pathologies of adult seen in Family Health Strategies (FHS). Was carried out a cross-sectional, observational and quantitative approach. The research instruments were anthropometric evaluation (weight, height and waist circumference) and the application of two questionnaires, one about socioeconomic and clinical data and another one about food consumption. The data were analyzed using SPSS software version 25.0 and the variables were related through the Pearson Chi-square test. We evaluated 180 patients of both sexes, of these $29.4 \%$ were overweight and $42.8 \%$ were obese, there was a prevalence of chronic diseases, mainly systemic arterial hypertension (SAH) and a high tendency to consume sweet foods. There was a
\end{abstract}


significant association among the nutritional diagnosis of obesity and systemic arterial hypertension, diabetes mellitus, dyslipidemia, depression, abdominal adiposity and use of medications. There was no significant association among dietary intake and nutritional status. The study showed high prevalence of overweight and obesity and chronic non-communicable diseases and consumption of sweet ultra-processed foods, being necessary strategies to coping these comorbidities.

Keywords: Nutritional Status. Family Health Strategy. Overweight. Obesity. Chronic Non-Communicable Diseases.

\section{INTRODUÇÃO}

O estado nutricional é determinado pelo equilíbrio entre o consumo de nutrientes e o gasto energético do organismo para suprir as necessidades de cada indivíduo ${ }^{1}$. 0 estado nutricional possui importante função para a qualidade de vida e de saúde. Quando inadequado, está relacionado ao desenvolvimento de patologias ${ }^{2}$. As doenças crônicas não transmissíveis (DCNT), como a diabetes mellitus tipo 2 (DM2), a hipertensão arterial sistêmica (HAS) e a obesidade encontram-se entre as principais causas de mortes no mundo e estão associadas aos hábitos alimentares e ao estado nutricional inadequado ${ }^{3}$.

A obesidade é definida como excesso de gordura corporal, resultante do desequilíbrio crônico entre consumo alimentar e gasto energético ${ }^{4}$. A prevalência de indivíduos com essa patologia cresce a cada ano. Indivíduos com excesso de peso (sobrepeso e obesidade) somam $86,2 \%$ da população adulta brasileira 5 . 0 excesso de peso está diretamente relacionado às DCNT, sendo um dos principais fatores de risco para a HAS, a DM2 e doenças cardiovasculares $(\mathrm{DCV})^{6}$, que estão entre as principais causas de mortes no mundo, contribuindo para o aumento de óbitos prematuros e para a diminuição da qualidade de vida³.

O Brasil atravessa um período de transição epidemiológica, com uma modificação dos padrões de saúde e doença, ocorrendo um aumento na incidência de obesidade em detrimento dos casos de desnutrição ${ }^{7}$. O Ministério da Saúde desenvolveu diversos programas e ferramentas, para controle e prevenção dos agravos, entre eles, o Sistema de Vigilância Alimentar e Nutricional (SISVAN), que tem como objetivo principal monitorar o padrão alimentar e o estado nutricional da população atendida pelo Sistema Único de Saúde (SUS) ${ }^{8}$, fornecendo subsídios para orientação e promoção de uma alimentação apropriada e saudável. 
A Atenção Básica à Saúde deve ser a principal porta de atendimento à população ${ }^{8}$. A Estratégia de Saúde da Família (ESF), através de uma equipe multidisciplinar, atende uma população definida, criando vínculo com uma área adscrita9 ${ }^{9}$ Essa equipe deve identificar o estado nutricional e o padrão alimentar dos indivíduos, a fim de detectar possíveis problemas nutricionais e estabelecer estratégias de enfrentamento, com o intuito de controlar e prevenir o excesso de peso e as DCNT e promover uma alimentação adequada e saudável ${ }^{8,10}$.

Diante disso, este estudo tem como objetivos avaliar o estado nutricional de adultos atendidos em Estratégias de Saúde da Família, identificar as patologias mais prevalentes, avaliar o consumo alimentar e relacionar essas variáveis com o estado nutricional.

\section{MÉTODO}

Trata-se de um estudo com delineamento transversal, observacional, de abordagem quantitativa. Participaram da investigação indivíduos de ambos os sexos com idade entre 20 e 59 anos, atendidos em cinco Estratégias de Saúde da Família (ESF) em uma cidade do Vale do Paranhana - RS/Brasil. A amostra foi aleatória por conveniência.

Os indivíduos foram abordados na sala de espera e convidados a participar da pesquisa mediante assinatura do termo de consentimento livre e esclarecido (TCLE). A coleta dos dados ocorreu entre os meses de agosto e setembro de 2018.

Para a coleta de dados, foram utilizados dois questionários, com questões estruturadas, conduzidos pela pesquisadora responsável, um incluindo dados socioeconômicos e clínicos e outro sobre o consumo alimentar, para a qual foi utilizado o formulário de marcadores de consumo alimentar do SISVAN $^{8}$ que avalia os alimentos consumidos no dia anterior à entrevista e comportamentos realizados durante as refeições. Todas as questões foram respondidas pelos participantes, sem acesso aos prontuários.

Além disso, realizou-se avaliação antropométrica. Os dados antropométricos (peso, altura e circunferência da cintura) foram coletados e classificados conforme o protocolo estabelecido pelo SISVAN ${ }^{1}$. O peso foi aferido utilizando balança digital da marca Avanutri ${ }^{\circledR}$ com capacidade máxima de $180 \mathrm{Kg}$. O paciente estava com o mínimo de roupa possível, descalço, ereto, com os pés juntos e os braços estendidos ao longo do corpo. Para a aferição da altura, utilizou-se estadiômetro portátil, marca 
Avanutri ${ }^{\circledR}$ com altura máxima de $210 \mathrm{~cm}$. O voluntário estava descalço, de pé, ereto, imóvel, com os braços esticados ao longo do corpo e com a cabeça orientada no plano horizontal. Por meio do peso e da altura obteve-se o Índice de Massa Corporal (IMC). Além disso, foi classificado o estado nutricional, conforme a recomendação da Organização Mundial da Saúde (OMS) ${ }^{11}$. A medida da circunferência da cintura (CC) foi realizada a partir do ponto médio entre a crista ilíaca e a última costela, com uma fita antropométrica flexível e inelástica com 2 metros de extensão, da marca Cescorf ${ }^{\circledR}$. O paciente estava de pé, ereto, com o abdômen relaxado e com os braços estendidos ao longo do corpo. Os pontos de corte adotados para a classificação foram os estabelecidos pela $\mathrm{OMS}^{4}$.

Os dados coletados foram tabulados em uma planilha do Microsoft Office Excel ${ }^{\circledR} 2013$ e analisados através do software Statistical Package for the Social Science (SPSS) versão 25.0, utilizando estatística descritiva com tabelas de frequência e medidas de tendência central e dispersão. A associação entre as variáveis foi verificada através do teste Qui-quadrado de Pearson. O nível de significância foi considerado quando $\mathrm{p}$ $<0,05$ e foi adotado um intervalo de confiança de $95 \%$.

O presente estudo foi aprovado pela Secretária Municipal de Saúde do município onde ocorreu a pesquisa e pelo Comitê de Ética em Pesquisa (CEP), da Universidade Feevale, sob o parecer número 2.827.021. Todos os participantes incluídos assinaram o TCLE após serem informados sobre a natureza do estudo, tendo respaldados todos os preceitos éticos da Resolução nº 466/2012, do Conselho Nacional de Saúde ${ }^{12}$.

\section{RESULTADOS}

Foram avaliados 180 adultos, com idade média de 40,88 $\pm 11,3$ anos, sendo a maioria mulheres $70 \% \quad(n=126)$. Os resultados das variáveis sociodemográficas são apresentados na Tabela 1. A HAS foi a doença crônica mais frequente, acometendo $30 \%$ dos indivíduos. A maioria não utilizava medicação contínua 54,4\%. A média da CC foi de $92,04 \pm 15,2 \mathrm{~cm}$, sendo que $65,6 \%$ dos adultos avaliados apresentou obesidade abdominal, em sua maioria mulheres, 52\% ( $n=93)$. Essas informações estão descritas na Tabela 2. 
Tamiris Gottlieb, Claudia Winter | Estado nutricional de adultos atendidos em Estratégias de Saúde da Família de um município do Vale do Paranhana-RS

Tabela 1 - Características sociodemográficas dos participantes da pesquisa atendidos em ESF.

\begin{tabular}{|c|c|c|}
\hline Variável & $\mathbf{N}$ & $\%$ \\
\hline \multicolumn{3}{|l|}{ Sexo } \\
\hline Feminino & 126 & 70,0 \\
\hline Masculino & 54 & 30,0 \\
\hline \multicolumn{3}{|l|}{ Cor da Pele } \\
\hline Branca & 148 & 82,2 \\
\hline Preta & 4 & 2,2 \\
\hline Parda & 28 & 15,6 \\
\hline \multicolumn{3}{|l|}{ Idade } \\
\hline 20 a 29 anos & 33 & 18,0 \\
\hline 30 a 39 anos & 47 & 26,0 \\
\hline 40 a 49 anos & 48 & 27,0 \\
\hline 50 a 59 anos & 52 & 29,0 \\
\hline \multicolumn{3}{|l|}{ Estado Civil } \\
\hline Casado & 61 & 33,9 \\
\hline Viúvo & 9 & 5,0 \\
\hline Solteiro & 68 & 37,8 \\
\hline Separado & 15 & 8,3 \\
\hline Outro & 27 & 15,0 \\
\hline \multicolumn{3}{|l|}{ Trabalho } \\
\hline Sim & 130 & 72,2 \\
\hline Não & 50 & 27,8 \\
\hline \multicolumn{3}{|l|}{ Escolaridade } \\
\hline Analfabeto & 2 & 1,1 \\
\hline Fundamental Incompleto & 86 & 47,8 \\
\hline Fundamental Completo & 27 & 15,0 \\
\hline Médio Incompleto & 9 & 5,0 \\
\hline Médio Completo & 47 & 26,1 \\
\hline Superior Incompleto & 4 & 2,2 \\
\hline Superior Completo & 5 & 2,8 \\
\hline \multicolumn{3}{|c|}{ Renda Familiar (salários mínimos) } \\
\hline Até $1(\mathrm{R} \$ 954,00)$ & 36 & 20,0 \\
\hline 1 a $3(R \$ 954,00$ a $2.862,00)$ & 111 & 61,7 \\
\hline 3 a $5(\mathrm{R} \$ 2.863,00$ a $4.770,00)$ & 26 & 14,4 \\
\hline$>5(\mathrm{R} \$>4.770,00)$ & 7 & 3,9 \\
\hline
\end{tabular}

Tabela 2 - Perfil de saúde, estado nutricional e estilo de vida de participantes da pesquisa atendidos em ESF.

\begin{tabular}{lcc}
\hline Variável & N & $\%$ \\
\hline DCNT & & \\
Hipertensão Arterial Sistêmica & 54 & 30,0 \\
Diabetes Mellitus & 15 & 8,3 \\
Dislipidemia & 14 & 7,8 \\
Depressão & 20 & 11,1 \\
Outras & 28 & 15,6 \\
Uso de Medicações & & \\
Sim & 82 & 45,6 \\
Não & 98 & 54,4 \\
\hline & & Continua
\end{tabular}




\begin{tabular}{lcc} 
Continuação da tabela 2 & & \\
\hline Variável & N & $\%$ \\
\hline Estado Nutricional & 1 & \\
$\quad$ Baixo Peso & 49 & 27,6 \\
Eutrofia & 53 & 29,4 \\
Sobrepeso & 77 & 42,8 \\
Obesidade & & \\
Circunferência da Cintura & 62 & 34,4 \\
Sem risco & 118 & 65,6 \\
Com risco & & \\
Estilo de Vida & 23 & 12,8 \\
$\quad$ Tabagismo & 3 & 1,7 \\
Bebida Alcoólica & 31 & 22,8 \\
Exercício Físico & 41 \\
\hline
\end{tabular}

Dos indivíduos com CC com risco, 81,4\% (n=69) apresentaram alguma patologia associada. Entre os adultos avaliados 100\% (n=15) dos que referiram DM, também apresentam adiposidade abdominal, os usuários com depressão somaram $85 \%$ $(n=17)$, dislipidemia $86 \% \quad(n=12)$ e dos que referiram HAS 79,6\% $(n=43)$ apresentaram CC acima da recomendação.

O IMC médio dos participantes foi de $29,88 \pm 7,05 \mathrm{~kg} / \mathrm{m}^{2}$, dentre esses, $29,4 \%(\mathrm{n}=53)$ estava com sobrepeso e 42,8\% ( $n=77)$ com obesidade, totalizando $72,2 \%(n=130)$ da amostra com excesso de peso (Tabela 2). A obesidade e o sobrepeso, se concentram em sua maioria, nas mulheres $75 \%$, porém, o sexo não teve associação significativa com o estado nutricional. Houve associação significativa entre o diagnóstico nutricional de obesidade e HAS, DM2, dislipidemias e depressão, com o uso de medicações, CC com risco e tabagismo, como pode ser visualizado na Tabela 3.

Tabela 3 - Relação entre estado nutricional e doenças crônicas em participantes da pesquisa atendidos em ESF

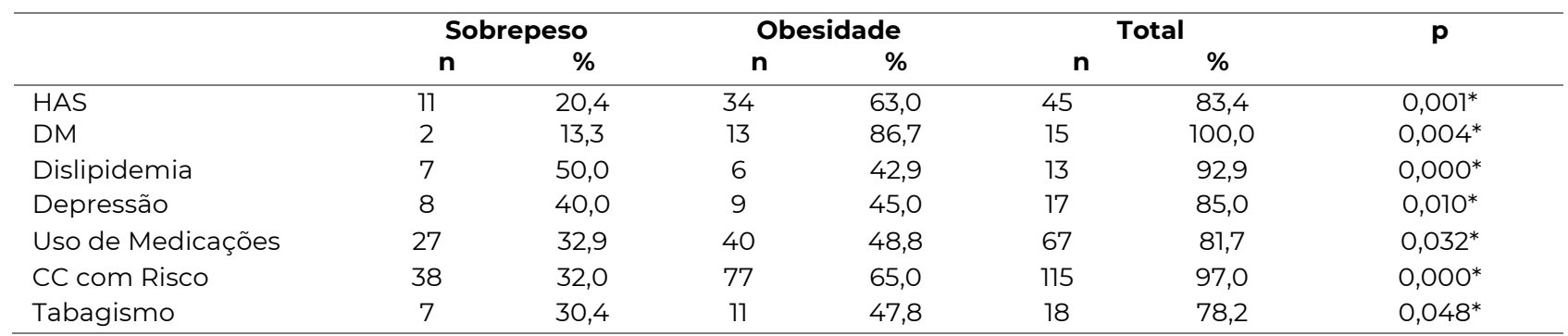

HAS: Hipertensão Arterial Sistêmica; DM: Diabetes Melittus; CC: Circunferência da Cintura; * $p<0,05$ Teste Qui-quadrado de Pearson.

Entre os indivíduos com DM2, 57\% $(\mathrm{n}=8)$, relataram ter dislipidemia associada e $93,3 \%(n=14)$ dos adultos com DM2, também tem HAS. Entre os indivíduos que referiram ser tabagistas, $52,2 \% \quad(n=12)$ apresentam patologias associadas, principalmente HAS, $58,3 \%(n=7)$. 
Através dos marcadores de consumo alimentar do SISVAN, foram avaliados os alimentos consumidos no dia anterior à entrevista. Não houve associação significativa entre o consumo alimentar e o estado nutricional dos indivíduos avaliados, porém destaca-se a elevada ingestão de bebidas adoçadas por adultos obesos $(p=0,051)$. Esses resultados podem ser visualizados na Tabela 4. Os indivíduos apresentaram bom consumo de feijão (65\%), frutas frescas $(68,3 \%)$ e verduras $(74,4 \%)$.

Tabela 4 - Relação entre o consumo alimentar e o excesso de peso em participantes da pesquisa atendidos em ESF, conforme formulário do SISVAN

\begin{tabular}{|c|c|c|c|c|c|}
\hline \multirow[b]{2}{*}{ Variáveis } & \multicolumn{2}{|c|}{ Sobrepeso } & \multicolumn{2}{|c|}{ Obesidade } & \multirow[b]{2}{*}{$\mathbf{p}$} \\
\hline & $\mathbf{n}$ & $\%$ & $\mathbf{N}$ & $\%$ & \\
\hline $\begin{array}{l}\text { Você tem costume de realizar as refeições assistindo à TV, } \\
\text { mexendo no computador e/ou no celular? }\end{array}$ & 26 & 29,9 & 36 & 41,4 & 0,761 \\
\hline Feijão & 36 & 30,8 & 48 & 41 & 0,491 \\
\hline Frutas frescas & 38 & 30,9 & 55 & 44,7 & 0,395 \\
\hline Verduras e/ou legumes & 40 & 29,9 & 57 & 42,5 & 0,939 \\
\hline Hambúrguer e/ou embutidos & 14 & 26,9 & 21 & 40,4 & 0,383 \\
\hline Bebidas adoçadas & 17 & 20 & 41 & 48,2 & 0,051 \\
\hline $\begin{array}{l}\text { Macarrão instantâneo, salgadinhos de pacote ou biscoitos } \\
\text { salgados }\end{array}$ & 9 & 31 & 10 & 34,5 & 0,695 \\
\hline Biscoito recheado, doces ou guloseimas & 20 & 28,6 & 24 & 34,3 & 0,081 \\
\hline
\end{tabular}

\section{DISCUSSÃO}

Neste estudo, observou-se maior prevalência da população feminina, o que condiz com outras pesquisas ${ }^{9,13,14}$ que também tiveram uma maior participação de mulheres. Isso pode ser justificado pelo fato de o público feminino ter uma maior preocupação com a saúde e por isso procurar mais por atendimento. O nível de escolaridade foi baixo, a maioria não completou o Ensino Fundamental. Esses dados corroboram os encontrados em pesquisas nacionais, que mostram que, quanto menor o nível de instrução, mais frequentes são as doenças crônicas e mais inadequado é o estado nutricional ${ }^{7,15}$.

As prevalências de sobrepeso e de obesidade da população adulta avaliada encontram-se elevados. Esse dado é condizente com outros estudos regionais, que mostram que mais da metade dos indivíduos adultos avaliados estão acima do peso $^{6,16,17}$, e com pesquisas de base nacional, como o Sistema de Vigilância de Fatores de Risco e Proteção para Doenças Crônicas por Inquérito Telefônico (VIGITEL) 2019, segundo o qual mais da metade da população adulta brasileira está acima do peso recomendado, e $19,5 \%$ dos homens e $21 \%$ das mulheres estão obesos ${ }^{15}$. O SISVAN Web de 2020 aponta que, na região Sul do país, 74,2\% das pessoas adultas avaliadas 
estão acima do peso. Esses dados são semelhantes com os do Brasil, onde o percentual da população com excesso de peso (sobrepeso e obesidade) alcança $66,74 \%$ e apenas $31,2 \%$ dos indivíduos são eutróficos ${ }^{18}$.

O percentual de indivíduos que apresentaram déficit de peso, na última Pesquisa Nacional de Saúde (PNS), foi significativamente baixo (1,6\%), o que caracteriza a transição nutricional que está ocorrendo no Brasil, com a diminuição dos casos de desnutrição e o aumento nos casos de sobrepeso e de obesidade em adultos, que representam 60,3\% e 25,9\%, respectivamente ${ }^{5}$. Esses dados são semelhantes aos apresentados na Pesquisa de Orçamentos Familiares (POF), segundo a qual $49 \%$ da população adulta tem excesso de peso e $14,8 \%$ é obesa. Ao estratificar esses percentuais por região, os estados da região Sul do país tiveram a maior prevalência ${ }^{19}$. Em ambos os estudos (PNS e POF), as mulheres apresentaram maior prevalência de excesso de peso (sobrepeso e obesidade), resultados que são corroborados por este estudo, o qual demonstrou que $75 \%$ das mulheres estão acima do peso.

A POF avaliou a adiposidade abdominal por meio da CC. Essa aferição mostrou que $37,7 \%$ dos indivíduos estavam com a medida aumentada ${ }^{19}$, o que representa fator de risco para DCV, DM, dislipidemias e síndrome metabólica, mesmo na ausência da obesidade generalizada ${ }^{7,20,21}$. A CC foi maior no sexo feminino, assim como neste estudo, em que $52 \%$ das mulheres estão com obesidade abdominal. A média da CC encontrada na população estudada foi de $92,04 \pm 15,2 \mathrm{~cm}$, sendo que mais da metade dos indivíduos avaliados apresentou obesidade abdominal. Resultado semelhante a outras pesquisas, como a de Linhares et al. ${ }^{22}$ e Pinho et al. ${ }^{23}$, onde 59,6\% ( $\left.n=850\right)$ e $69,9 \%(n=640)$ das mulheres apresentaram adiposidade abdominal. Ao relacionar a CC com as DCNT avaliadas nesta pesquisa, verificou-se elevada frequência de CC elevada associada a doenças crônicas, todos os participantes que referiram DM apresentam obesidade abdominal, além disso, indivíduos com depressão, dislipidemia e HAS apresentaram CC acima da recomendação, com risco elevado para complicações metabólicas, DCV e eventos coronarianos ${ }^{24-27}$.

O acúmulo de gordura na região abdominal e o excesso de peso são uns dos principais fatores de risco para a $\mathrm{HAS}^{28}$, doença multifatorial caracterizada por elevação sustentada dos níveis pressóricos ${ }^{26}$. No Brasil, atinge 32,5\% (36 milhões) dos indivíduos adultos e mais de $60 \%$ dos idosos contribuindo direta ou indiretamente para $50 \%$ das mortes por DCV 26,27 . Na pesquisa do VIGITEL (2019), 24,5\% dos 
entrevistados referiram diagnóstico de $\mathrm{HAS}^{15}$, mantendo se estável em relação à pesquisa do ano anterior, onde $24,7 \%$ auto referiram HAS. Dos participantes que referiram HAS neste estudo, 83,4\% ( $n=45)$ apresentam sobrepeso ou obesidade, prevalência semelhante à encontrada em um estudo com hipertensos realizado no Maranhão, de acordo com o qual 75,7\% dos indivíduos apresentavam sobrepeso ou obesidade $^{28}$. Outras pesquisas também são corroboradas por este achado, como os estudos de Chagas e Almeida ${ }^{14}$, Coqueiro et al. ${ }^{29}$, Silva et al. ${ }^{30}$ e Serra et al. ${ }^{28}$ que encontraram $34,7 \%, 43 \%, 71 \%$ e $75,7 \%$, respectivamente, dos hipertensos avaliados com sobrepeso ou com obesidade.

Uma das doenças associada à HAS e ao excesso de peso é o DM2, que pode corresponder a até $95 \%$ de todos os casos de DM, possuindo etiologia complexa e multifatorial, que envolve fatores genéticos e ambientais ${ }^{26,27}$. Dos indivíduos entrevistados na pesquisa do VIGITEL ${ }^{15}, 7,4 \%$ auto referiram DM. Em relação à pesquisa do ano anterior, esse dado, manteve-se estável, apresentando uma leve redução de $0,3 \%$. Considerando as pesquisas realizadas pelo VIGITEL nos anos anteriores, a DM apresenta uma tendência de evolução desfavorável, com aumento da prevalência ao longo das pesquisas. Dos participantes que referiram DM2 neste estudo, $100 \%$ apresentam sobrepeso ou obesidade. Em estudos de Maccarone et al. ${ }^{31}$, Lima et al. ${ }^{32}$ Marinho et al. ${ }^{33}$ foram encontrados resultados inferiores; onde 80,6\%, $73,6 \%$ e $59,7 \%$ dos diabéticos avaliados estavam com o peso acima do recomendado. O excesso de peso e a DM podem estar associadas a fatores como a inatividade física e o consumo alimentar inadequado ${ }^{26,27}$. A associação entre DM, HAS e dislipidemia são considerados fatores de risco para o desenvolvimento da $\mathrm{DCV}^{34}$. $\mathrm{O}$ paciente com DM2 tem maior risco de desenvolver dislipidemia devido a alterações no metabolismo das lipoproteínas circulantes ${ }^{34}$. Nesta pesquisa, mais da metade dos indivíduos relataram ter hiperlipidemia e DM2 associadas. Assunção et al. ${ }^{35}$ encontrou resultados semelhantes em diabéticos, onde, 57,2\% estavam com os níveis de lipídeos elevados.

A estudo de base populacional de Valença et $\mathrm{al}^{36}$, avaliou 884 adultos, a dislipidemia foi verificada através de exames bioquímicos, verificando-se que $64,2 \%$ da amostra possuía níveis elevados de lipídeos. Na presente pesquisa, apenas uma pequena parcela dos entrevistados relatou diagnóstico de dislipidemia, o que pode ser justificado pelo desconhecimento da patologia pelos participantes, visto que o diagnóstico da mesma, requer exames laboratoriais. $\mathrm{N}$ presente pesquisa, desses indivíduos, porém, apenas um não possui sobrepeso ou obesidade, havendo 
associação significativa entre o diagnóstico de dislipidemias e o estado nutricional. Similar a Garcez et al. onde $73 \%$ dos indivíduos com hiperlipidemias apresentavam excesso de peso ${ }^{37}$.

Uma das doenças mais referida pelos participantes avaliados foi a depressão, patologia caracterizada por um conjunto de sintomas que afetam a área afetiva e emocional. A OMS estima que mais de 300 milhões de pessoas no mundo vivam com depressão, no Brasil 11,5 milhões de indivíduos são acometidos por essa afecção ${ }^{38}$. Nesta pesquisa, dos adultos que referiram depressão, mais da metade estão com sobrepeso ou obesidade. Indivíduos com depressão e obesidade apresentam sintomas como ansiedade, baixa autoestima, redução da energia, mudanças no apetite e diminuição das atividades ${ }^{38}$, o que pode estar associado, ainda, a uma ingestão excessiva de calorias e a um baixo gasto energético, fatores que favorecem o acúmulo de gordura, o que justificaria o perfil encontrado nesta pesquisa. Indivíduos obesos, apresentam tendência ao desenvolvimento de depressão ${ }^{39,40}$. A associação estatística entre obesidade e uso de medicações pode ser explicada pela alta prevalência de doenças crônicas no grupo de indivíduos avaliados ${ }^{41}$.

A maior parte da população estudada declarou não realizar atividades físicas, apesar de não ter ocorrido correlação significativa entre inatividade física e obesidade. Todavia, indivíduos menos ativos ou sedentários apresentam risco cardiovascular aumentado ${ }^{42}$. Esses dados são semelhantes aos encontrados em uma pesquisa que avaliou 138 adultos, a qual mostrou que a maior parte da amostra $(64,49 \%)$ referiu não praticar qualquer tipo de atividade física, sendo, portanto, sedentário ${ }^{42}$. A prática regular de exercícios físicos ou de esportes é considerada fator de proteção à saúde, $46 \%$ da população brasileira não alcança um nível suficiente de atividade física $^{15}$. A introdução dos exercícios físicos na rotina é fator protetor, assim como faz parte do tratamento das DCNT, promovendo melhora na qualidade de vida ${ }^{43}$.

Uma pequena parcela dos indivíduos entrevistados relatou tabagismo. Porém, ao relacionar o tabagismo com o estado nutricional, encontra-se correlação significativa entre obesidade e tabagismo sendo que $78 \%$ dos fumantes têm sobrepeso ou obesidade. Em uma pesquisa que avaliou 52 indivíduos atendidos em ESF, $21 \%(n=10)$ dos participantes relataram fumar e, assim como neste estudo, ao analisar o estado nutricional, a metade desses indivíduos tinha excesso de peso ${ }^{43}$. 0 uso de cigarros é o principal fator do surgimento de diversas doenças crônicas, sendo considerado a principal causa de morte evitável em todo o mundo. Nesta pesquisa, 
mais da metade dos indivíduos fumantes, apresentam patologias associadas, principalmente HAS.

Outro parâmetro nutricional avaliado neste estudo foi o consumo alimentar, que foi mensurado por meio do questionário de marcadores de consumo alimentar do SISVAN $^{1}$. O formulário apresenta como característica a avaliação dos alimentos consumidos no dia anterior, o que ameniza possíveis vieses de memória. Porém, podem não representar o padrão alimentar da população, além de existir a possibilidade de omissão de informações por parte do informante ${ }^{1}$. Esta pesquisa não encontrou associação significativa entre o consumo alimentar e o estado nutricional dos indivíduos avaliados, destaca-se, porém, a elevada ingestão de bebidas adoçadas por adultos com obesidade, o que pode contribuir para a elevada prevalência de excesso de peso encontrada. A OMS e o Ministério da Saúde recomendam que o consumo de açúcar seja inferior a 10\% do valor energético total 44 . Na POF (2020), 85,4\% dos indivíduos relataram adoçar alimentos ou bebidas, sendo que o açúcar representa 5,8\% das calorias diárias ${ }^{45}$. A ingestão elevada de calorias favorece $o$ acúmulo de gordura.

O padrão alimentar da população brasileira é caracterizado pelo consumo habitual de arroz e feijão, juntamente com um elevado consumo de alimentos de alto valor energético e de baixo valor nutricional ${ }^{45}$. Esse padrão representa a transição do consumo nutricional, na qual a escassez de alimentos vem sendo substituída pelo excesso de alimentos pouco nutritivos ${ }^{9}$. Esse perfil influencia fortemente a ocorrência do excesso de peso, da $\mathrm{HAS} \mathrm{DM}^{46}$. Nesta pesquisa, o consumo de feijão foi relatado pela maioria dos participantes. Na PNS, o consumo regular desse alimento foi relatado por $68,3 \%$ das pessoas ${ }^{47}$ e, no VIGITEL, por $59,7 \%{ }^{15}$. Observase uma tendência desfavorável no consumo de feijão, conforme estes estudos. $O$ feijão fornece nutrientes importantes, sendo o seu consumo adequado um fator protetor para doenças.

Apesar de grande parte dos entrevistados terem relatado o consumo de frutas, verduras ou legumes no dia anterior ao da entrevista, a quantidade ingerida não foi avaliada. Dessa maneira, pode haver um déficit na ingestão desses alimentos, visto que o recomendado pela OMS são 400 gramas diários, o que equivale a cinco porções ao dia ${ }^{48}$. Na PNS, apenas $13 \%$ da população entrevistada consumia essa quantidade ${ }^{47}$. No VIGITEL, o consumo recomendado de frutas e hortaliças foi de $22,9 \%{ }^{15}$, apresentando uma tendência favorável de consumo nos últimos anos, 
principalmente entre as mulheres. A alimentação inadequada é um dos fatores de risco para o desenvolvimento ou para o agravamento das DCNT, como a HAS, a DM e as dislipidemia, patologias que apresentaram grande prevalência nesta pesquisa e que estão diretamente associadas à obesidade.

Outra mudança nos padrões que ocorreu nos últimos anos e que contribui para a tendência de aumento na obesidade, foi a introdução dos meios eletrônicos na sociedade. Quase metade dos entrevistados relatou realizar as refeições assistindo à televisão ou mexendo no celular. A desatenção praticada durante a alimentação está associada à obesidade. Essa prática pode fazer com que os indivíduos comam além de suas necessidades, pois não percebem seus atos. Comer com atenção pode auxiliar na melhoria dos hábitos alimentares bem como no controle da obesidade ${ }^{49}$.

Dessa maneira, estratégias de educação alimentar e nutricional, que promovam o conhecimento sobre uma alimentação saudável, devem estar inseridas na atenção básica, proporcionando melhora na qualidade de vida e a prevenção de agravos à saúde dos indivíduos.

Algumas limitações nessa pesquisa podem ser ressaltadas, como o delineamento transversal, o questionário de marcadores de consumo alimentar, que avalia apenas o dia anterior, o que pode não refletir a ingestão habitual de maneira fidedigna, além disso, as patologias podem ter sido sub relatadas, devido ao desconhecimento dos entrevistados sobre as mesmas.

\section{CONCLUSÃO}

O estudo demonstrou que o estado nutricional da maioria dos adultos atendidos em ESF está acima do recomendado. Conclui-se que a população estudada está com excesso de peso (sobrepeso e obesidade), elevada prevalência de doenças crônicas e tendência ao consumo de alimentos ultraprocessados doces, sendo necessário estabelecer estratégias de enfrentamento, de manejo e de promoção de hábitos saudáveis, visando ao controle de peso, mudanças nos hábitos de vida e redução do sobrepeso e obesidade. Mais estudos são necessários, a fim de avaliar as variáveis que contribuem para o estado nutricional inadequado mais detalhadamente.

\section{REFERÊNCIAS}

1. Brasil. Ministério da Saúde (MS). Orientações para a coleta e análise de dados antropométricos em serviços de saúde: Norma Técnica do Sistema de Vigilância Alimentar e Nutricional - SISVAN. Brasília: MS; 2011. 
2. Pereira IFDS, Spyrides MHC, Andrade LDMB. Estado nutricional de idosos no Brasil: uma abordagem multinível. Cad Saude Publica 2016; 32(5):e00178814.

3. Brasil. Ministério da Saúde (MS). Plano de ações estratégicas para o enfrentamento das doenças crônicas não transmissíveis (DCNT) no Brasil 2011-2022. Brasília: MS; 2011.

4. World Health Organization (WHO). Obesity: preventing and managing the global epidemic: report of a WHO Expert Consultation on Obesity. Geneva: WHO; 2000.

5. Instituto Brasileiro de Geografia e Estatística (IBGE). Pesquisa nacional de saúde: 2019: Atenção primária à saúde e informações antropométricas. Rio de Janeiro: IBGE; 2020.

6. Barroso TA, Marins LB, Alves R, Gonçalves ACS, Barroso SG, Rocha GDS. Associação Entre a Obesidade Central e a Incidência de Doenças e Fatores de Risco Cardiovascular. International Journal of Cardiovascular Sciences 2017; 30(5):416-424.

7. Instituto Brasileiro de Geografia e Estatística (IBGE). Pesquisa nacional de saúde: 2013: indicadores de saúde e mercado de trabalho: Brasil e grandes regiões. Rio de Janeiro: IBGE; 2016.

8. Brasil. Ministério da Saúde (MS). Orientações para avaliação de marcadores de consumo alimentar na atenção básica. Brasília: MS; 2015.

9. Silvani J, Buss C, Pena GDG, Recchi AF, Wendland EM. Consumo alimentar de usuários do Sistema Único de Saúde segundo o tipo de assistência e participação no Bolsa Família. Cien Saude Colet 2018; 23(8):2599-2608.

10. Paiva, ALS. O nutricionista e o cuidado ao indivíduo com excesso de peso na atenção básica do Distrito Federal [Dissertação]. Brasília (DF): Universidade de Brasília; 2016.

11. World Health Organization (WHO). Physical Status: the use and interpretation of anthropometry. Geneva, Switzerland: WHO; 1995. (WHO Technical Report Series, n. 854).

12. Brasil. Resolução $n^{0} 466$, de 12 de dezembro de 2012. Aprova as diretrizes e normas regulamentadoras de pesquisa envolvendo seres humanos. Diário Oficial da União 2012; 12 dez.

13. Pinho CPS, Diniz AS, Arruda IKG, Filho MB, Coelho PC, Sequeira LAS, Lira PIC. Prevalência e fatores associados à obesidade abdominal em indivíduos na faixa etária de 25 a 59 anos do Estado de Pernambuco, Brasil. Cad Saude Publica 2013; 29(2):313-324.

14. Chagas JAS, Almeida ANF. Perfil Epidemiológico e Qualidade de Vida de Hipertensos Usuários de uma Unidade Básica De Saúde De Macapá, Amapá. Cad. Edu Saúde e Fis 2016; 3(6).

15. Brasil. Ministério da Saúde (MS). Vigitel Brasil 2019: vigilância de fatores de risco e proteção para doenças crônicas por inquérito telefônico. Brasília: MS; 2020.

16. Barbosa AM, Lacerda DAL. Associação entre Consumo Alimentar e Estado Nutricional em Agentes Comunitários de Saúde. R Bras Ci Saúde 2017; 21(3):189196.

17. Carvalho EV, Costa VVL, Araújo MS, Martens IBG, Sá NNB, Silva RA. Correlação entre o estado nutricional e o risco coronariano de adultos atendidos em uma 
Ação educativa em Belém Pará. RBONE-Revista Brasileira de Obesidade, Nutrição e Emagrecimento 2016; 10(55):40-49.

18. Brasil. Ministério da Saúde (MS). Banco de dados do Sistema Único de SaúdeDATASUS; 2020. [acessado 2020 dez 25]. Disponível em: http://dabsistemas.saude.gov.br/sistemas/sisvanV2/relatoriopublico/index.

19. Instituto Brasileiro de Geografia e Estatística (IBGE). Pesquisa de Orçamentos Familiares 2008-2009: antropometria e estado nutricional de crianças, adolescentes e adultos no Brasil. Rio de Janeiro: IBGE; 2010.

20. Araújo LM, Lima B, Dantas GF, Lins PRM. Excesso de peso e risco cardiovascular em trabalhadores da construção civil. BRASPEN J 2016; 31(4):283-287

21. Machado SP, Rodrigues DGC, Viana KDAL, Sampaio HAC. Correlação entre o índice de massa corporal e indicadores antropométricos de obesidade abdominal em portadores de diabetes mellitus tipo 2. Rev Bras Promoç Saúde 2013; 25(4):512-520.

22. Linhares RS, Horta BL, Gigante DP, Dias-da-Costa JS, Olinto MTA. Distribuição da obesidade geral e abdominal em adultos de uma cidade do sul do Brasil. Cad Saude Publica 2012; 28(3):438-448.

23. Pinho CPS, Diniz AS, Arruda IKG, Filho MB, Coelho PC, Sequeira LAS, Lira PIC. Prevalência e fatores associados à obesidade abdominal em indivíduos na faixa etária de 25 a 59 anos do Estado de Pernambuco, Brasil. Cad Saude Publica 2013; 29(2):313-324.

24. Brasil. Associação Brasileira para o Estudo da Obesidade e da Síndrome Metabólica (ABESO). Diretrizes brasileiras de obesidade. São Paulo: SP; 2016.

25. Sociedade Brasileira de Diabetes. Diretrizes da Sociedade Brasileira de Diabetes 2019-2020. São Paulo: SP; 2011.

26. Sociedade Brasileira de Cardiologia. $7^{\text {a }}$ Diretriz Brasileira de Hipertensão Arterial. Arquivos Brasileiros de Cardiologia. Rio de Janeiro: RJ; 2016.

27. Wharton, Sean et al. Obesity in adults: a clinical practice guideline. CMAJ, 2020.

28. Serra MM, Pereira LCO, Fontenele DF, Viveiros MTM, Lima RA. Condições clínicas e antropométricas de hipertensos atendidos em um centro de saúde de São Luís, MA. Rev Pesq Saúde 2015; 16(2):107-111.

29. Coqueiro RS, Nery AA, Cruz ZV, Sá CKC. Fatores associados ao sobrepeso em adultos acompanhados por uma unidade de saúde da família. Rev. bras. cineantropom. desempenho hum. 2008; 10(2):149-154.

30. Silva GN, Gonzales MF, Silveira FM, Girata MY, Abrão LAA, Oda JMM. Hipertensão e obesidade dos indivíduos inseridos no programa hiperdia no município de Três Lagoas, MS. Rev. Gestão e Saúde 2014; 5(1):2641-2646.

31. Maccarone SD, Lima DB, Ferreira EB. Rastreamento da síndrome metabólica e qualidade de vida dos diabéticos adscritos a uma unidade de Estratégia de Saúde da Família em um município do sul de Minas Gerais. Rev. Aten. Saúde 2017; 15(51):98-105.

32. Lima RF, Fontbonne A, Carvalho EMF, Montarroyos UR, Barreto MNSC, Cesse EAP. Fatores associados ao controle glicêmico em pessoas com diabetes na Estratégia Saúde da Família em Pernambuco. Rev EsC Enferm USP 2016; 50(6):937945. 
33. Marinho NBP, Vasconcelos HCA, Alencar AMPG, Almeida PC, Damasceno MMC. Diabetes mellitus: fatores associados entre usuários da estratégia saúde da família. Acta Paul Enfer. 2012; 25(4):595-600.

34. Pinho L, Aguiar APS, Oliveira MR, Barreto NAP, Ferreira CMM. Hipertensão e dislipidemia em pacientes diabetes mellitus tipo 2: uma revisão integrativa. Renome 2015; 4(1):87-101.

35. Assunção SC, Fonseca AP, Silveira MF, Caldeira AP, Pinho L. Conhecimento e atitude de pacientes com diabetes mellitus da Atenção Primária à Saúde. Esc Anna Nery 2017; 21(4):1-7.

36. Valença SEO, et al. Prevalência de dislipidemias e consumo alimentar: um estudo de base populacional. Cien Saude Colet, 2020.

37. Garcez MR, Pereira JL, Fontanelli MM, Marchioni DML, Fisberg RM. Prevalência de dislipidemia segundo estado nutricional em amostra representativa de São Paulo. Arq Bras Cardiol 2014; 103(6):476-84.

38. Organização Panamericana de Saúde (OPAS). 2018. [acessado 2020 dez 24]. Disponível em: https://www.paho.org/bra/

39. Wannmacher L. Obesidade como fator de risco para morbidade e mortalidade: evidências sobre o manejo com medidas não medicamentosas. OPAS/OMSRepresentação Brasil 2016; 1(7):1-10.

40. World Health Organization (WHO). Guidelines for the management of physical health conditions in adults with severe mental disorders. Geneva: WHO; 2018.

41. Krebs PZ. Características e demandas dos usuários atendidos no serviço de acolhimento de nutrição em uma unidade básica de saúde. [Trabalho de Conclusão de Curso]. Porto Alegre (RS): Universidade Federal do Rio Grande do Sul; 2015.

42. Carvalho EV, Costa VVL, Araújo MS, Martens IBG, Sá NNB, Silva RA. Correlação entre o estado nutricional e o risco coronariano de adultos atendidos em uma Ação educativa em Belém Pará. RBONE-Revista Brasileira de Obesidade, Nutrição e Emagrecimento 2016; 10(55):40-49.

43. Sassi MM, Franco GP, Petroni S, Dalegrave D, Nogueira GM. Quantificação de risco para doenças cardiovasculares em usuários de uma Estratégia de Saúde da Família. Rev Enferm UFSM 2015; 5(2):204-212.

44. World Health Organization (WHO). Guideline: sugars intake for adults and children. Geneva: WHO; 2015.

45. Instituto Brasileiro de Geografia e Estatística (IBGE). Pesquisa de Orçamentos Familiares 2017-2018: análise do consumo alimentar pessoal no Brasil. Rio de Janeiro: IBGE; 2020.

46. Brasil. Ministério da Saúde (MS). Diretrizes do NASF. Núcleo de Apoio à Saúde da Família. Brasília: MS; 2009. Cadernos de Atenção Básica nº 27.

47. Instituto Brasileiro de Geografia e Estatística (IBGE). Pesquisa nacional de saúde: 2019: Percepção do Estado de Saúde, Estilo de Vida e Doenças Crônicas. Rio de Janeiro: IBGE; 2020.

48. World Health Organization (WHO). Diet, nutrition and the prevention of chronic diseases. Report of a joint WHO/FAO expert consultation. Geneva: WHO; 2003. 
Tamiris Gottlieb, Claudia Winter | Estado nutricional de adultos atendidos em Estratégias de Saúde da Família de um município do Vale do Paranhana-RS

49. Janssen LK, Duif I, Loon I, Vries JH, Speckens AE, Cools R, Aarts E. Greater mindful eating practice is associated with better reversal learning. Scientific reports 2018; 8(1): 5702.

Submissão: $19 / 08 / 2020$

Aprovação: 05/01/2021 